\title{
Burnout e depressão em residentes de um Programa Multiprofissional em Oncologia: estudo longitudinal prospectivo
}

\author{
Burnout and depression in residents of a Multi- \\ professional Program in Oncology: a longitudinal \\ prospective study
}

\author{
Ismar Lima Cavalcanti \\ Fernando Lopes Tavares de Lima $^{I}$ \\ Telma de Almeida Souza \\ Mario Jorge Sobreira da Silval
}

\author{
PALAVRAS-CHAVE \\ - Educação de Pós-Graduação \\ - Burnout \\ - Depressão \\ - Saúde do Trabalhador \\ - Oncologia.
}

\section{RESUMO}

A residência multiprofissional é uma modalidade de especialização lato sensu que se caracteriza pelo treinamento em serviço. A escassa experiência profissional prévia, a extensa carga horária do programa e o duplo papel do estudante-trabalhador podem levar o residente ao adoecimento. O objetivo deste estudo foi analisar a ocorrência de síndrome de burnout e de depressão entre residentes de um programa multiprofissional em oncologia. Trata-se de estudo prospectivo longitudinal com 46 residentes de um programa de residência multiprofissional em oncologia. Foram utilizados três instrumentos de coleta de informações: um para identificar características sociodemográficas e afastamentos por motivos de saúde; o Maslach Burnout Inventory; e, o Inventário de Depressão de Beck. Os instrumentos de coleta de dados foram aplicados, nos mesmos participantes do estudo, em 3 momentos ao longo de dois anos: no início, no final do primeiro e do segundo ano da residência. Os resultados referentes às características sociodemográficas, à intensidade das três dimensões do burnout, à frequência da síndrome de burnout e à frequência e intensidade da depressão foram analisados por estatística descritiva. O teste exato de Fisher foi aplicado para análise de correlação entre as variáveis relacionadas aos hábitos pessoais e aos afastamentos por motivos de saúde com a presença de síndrome de burnout ou de depressão. Os scores dos três aspectos do burnout e da depressão nos 3 momentos estudados foram comparados utilizando o teste de Wilcoxon. Ao final do estudo identificou-se que 75,0\% dos participantes apresentaram sindrome de burnout e 72,5\% tiveram algum nível de depressão. Quanto aos componentes do burnout, houve aumento dos scores de exaustão emocional e baixa realização pessoal. Também houve aumento da frequência e da intensidade de depressão. Observou-se correlação positiva entre afastamento por motivos de saúde e presença de burnout e entre ocorrência de síndrome de burnout e casos de depressão. A síndrome de burnout esteve correlacionada com ocorrência de depressão e ambos os problemas tiveram aumento significativo ao longo do programa de residência. Os achados apontam para a gravidade do problema, considerando que ambas as condições apareceram no primeiro ano de curso. Estratégias para prevenção e controle são necessárias, a fim de minimizar as consequências na aprendizagem, na qualidade de vida dos residentes e na assistência prestada aos pacientes com câncer. 


\section{KEY WORDS:}

- Education Graduate

- Burnout

- Professional

- Depression

- Occupational Health

- Medical Oncology.
ABSTRACT

The multi-professional residency is a modality of a lato sensu specialization course characterized by in-service training. The lack of previous professional experience, the extensive workload of the program and the dual role of the student-worker can lead the resident to illness. The aim of this study was to analyze the occurrence of burnout syndrome and depression among residents of a multi-professional program in oncology. This is a prospective longitudinal study with 46 residents of a multi-professional residency program in oncology. Three instruments of information collection were used: one to identify sociodemographic characteristics and absence for health reasons; Maslach Burnout Inventory; and Beck Depression Inventory. The data collection instruments were applied among the same participants of the study, in three instances over a two-year period: at the beginning, at the end of the first and of the second years of residence. The results regarding the sociodemographic characteristics, the intensity of the three dimensions of burnout, the frequency of burnout syndrome and the frequency and intensity of depression were analyzed by descriptive statistics. Fisher's exact test was applied to analyze the correlation between variables related to personal habits and leave of absence for health reasons with the presence of burnout syndrome or depression. The scores of the three aspects of burnout and depression in the three instances studied were compared using the Wilcoxon test. At the end of the study, it was identified that $75.0 \%$ of the participants presented burnout syndrome and $72.5 \%$ had some level of depression. As for the subscales of burnout, there was an increase in emotional exhaustion and in low personal accomplishment. There was also an increase in the frequency and intensity of depression. There was a positive correlation between leave of absence for health reasons and the presence of burnout and between the occurrence of burnout syndrome and cases of depression. Burnout syndrome was correlated to the occurrence of depression and both problems displayed significant increase throughout the residency program. The findings point to the severity of the problem, considering that both conditions appeared in the first year of the course. Strategies for prevention and control are necessary to minimize the consequences on learning, residents' quality of life and the care provided to cancer patients.

Recebido em 15/7/2017

Aceito em 20/10/2017

\section{INTRODUÇÃO}

Passados quase trinta anos de promulgação da Constituição Federal, diversos desafios para consolidação do Sistema Único de Saúde (SUS) ainda persistem, destacando-se a qualificação de profissionais que atendam às necessidades da população. O incentivo à ampliação de vagas em programas de residência multiprofissional em saúde tem sido uma das alternativas de enfrentamento a esse desafio ${ }^{1}$.

A residência multiprofissional é reconhecida como modalidade de ensino de pós-graduação lato sensu, sob forma de curso de especialização. É caracterizada por ensino em serviço, com carga horária de sessenta horas semanais e duração mínima de dois anos, em regime de dedicação exclusiva ${ }^{2}$. A escassa experiência profissional prévia, a extensa carga horária do programa e o duplo papel do estudante-trabalhador podem levar o residente ao adoecimento.
Dentre as condições atualmente preocupantes, encontra-se a síndrome de burnout, doença ocupacional caracterizada por despersonalização, exaustão emocional e baixa realização pessoal com o trabalho ${ }^{3,4}$. A síndrome ocorre em resposta ao estresse crônico, afetando a realização de tarefas e a produtividade, com resultado não apenas sobre os indivíduos, mas, também, sobre os relacionamentos interpessoais, sobre a instituição onde o processo se desenvolve e, por fim, sobre a sociedade ${ }^{5}$.

Profissionais da saúde são particularmente afetados ${ }^{6}$, sendo mais acometidos aqueles que lidam com câncer ${ }^{7,8}$. Estudos sinalizam, também, que durante os anos de treinamento em programas de residência a prevalência é elevada ${ }^{9,10}$.

Além do burnout, há também relatos de depressão em profissionais de saúde residentes. A depressão é um transtor- 
no mental comum, caracterizada pela presença de persistente tristeza ou perda de interesse ou de prazer, que pode ser acompanhada de diversos sintomas, tais como: baixa autoestima, cansaço, baixa concentração e dificuldade de tomar decisões ${ }^{11}$. Tanto a síndrome de burnout, quanto a depressão, prejudicam a vida profissional, interferem em situações externas ao ambiente profissional e podem comprometer o tratamento e a segurança dos pacientes ${ }^{9,12,13}$.

O objetivo deste estudo foi analisar a ocorrência de síndrome de burnout e de depressão entre residentes ao longo de um programa multiprofissional em oncologia.

\section{MÉTODO}

Trata-se de um estudo longitudinal e prospectivo realizado com os residentes admitidos e matriculados no ano de 2014 em um programa de residência multiprofissional de um centro de assistência de alta complexidade em oncologia do Rio de Janeiro. A coleta de dados foi realizada no início do programa (T1), ao término do primeiro (T2) e do segundo ano (T3). Foram incluídos todos aqueles que já se encontravam matriculados no primeiro momento, que correspondeu a primeira semana de aula. Tratou-se, portanto, de uma coorte fechada.

Foram utilizados três instrumentos de coleta de informações. O primeiro foi um questionário estruturado para identificar dados a respeito das características sociodemográficas (idade, gênero, estado civil, filhos, situação de moradia, principal meio de transporte, tempo de locomoção, categoria profissional e tempo de formado), hábitos pessoais (hobby, atividade física, tabagismo e consumo de álcool) e afastamento por motivo de saúde.

O segundo questionário foi o Maslach Burnout Inventory-Human Services Survey (MBI-HSS) traduzido e validado para o português ${ }^{14}$. Trata-se de um questionário de autopreenchimento composto por 22 itens e respondido por escala tipo Likert (0 - Nunca; 1 - Algumas vezes ao ano ou menos; 2 - Uma vez ao mês ou menos; 3 - Algumas vezes por mês; 4 - Uma vez por semana; 5 - Algumas vezes por semana; 6 - Todos os dias).

Este instrumento é utilizado para avaliar os três aspectos do burnout: exaustão emocional (EE), despersonalização (DE) e realização pessoal (RP). A subescala de EE (nove itens) avalia as queixas sobre sentir-se no limite e exausto pelo trabalho. A subescala de DE (cinco itens) mede respostas impessoais e falta de empatia durante a atividade profissional. A subescala de RP (oito itens) avalia os sentimentos de competência e conquista de sucesso no trabalho ${ }^{14}$.
Cada um destes aspectos é medido separadamente, o que resulta em três pontuações para cada participante. Essas pontuações, por subescala, foram analisadas utilizando-se os seguintes parâmetros: EE ( $\leq 18$ - Baixa; 19 a 26 - Moderada; $\geq 27$ - Alta); DE ( $\leq 5$ - Baixa; 6 a 9 - Moderada; $\geq 10$ - Alta) e RP ( $\geq 40$ - Baixa; 34 a 39 - Moderada; $\leq 33$ - Alta). A síndrome de burnout foi considerada presente quando altos níveis de EE e/ou DE foram encontrados ${ }^{15}$.

Para avaliação da depressão foi utilizado o inventário de depressão de Beck $^{16}$, constituído por 21 itens e validado em português ${ }^{17}$. Foram utilizados os seguintes pontos de corte: $<10$ - Sem depressão ou depressão mínima; 10 a 18 - Depressão leve a moderada; 19 a 29 - Depressão moderada a grave; 30 a 63 - Depressão grave. A depressão foi considerada presente quando encontrados os níveis correspondentes às classificações leve, moderada ou grave ${ }^{17}$.

Os resultados referentes às características sociodemográficas, à intensidade das três dimensões do burnout, à frequência da síndrome de burnout e à frequência e intensidade da depressão foram analisados por estatística descritiva.

O teste exato de Fisher foi aplicado para análise de correlação entre as variáveis relacionadas aos hábitos pessoais e aos afastamentos por motivos de saúde com a presença de síndrome de burnout ou de depressão. O teste foi utilizado, também, para identificar existência de relação entre síndrome de burnout e depressão, bem como, para verificar a diferença da frequência da síndrome de burnout e da depressão ao longo do tempo.

Os scores dos três aspectos do burnout e da depressão entre o início (T1), o final do primeiro (T2) e do segundo ano (T3) da residência foram comparados utilizando o teste de Wilcoxon.

O nível de significância foi considerado de 0,05 para ambos os testes. Para a análise estatística foram utilizados os programas de domínio público $\mathrm{R}$ versão 3.3.2 (The R Foundation for Statistical Computing, Viena, Áustria; http:// www.r-project.org) e Epi Info versão 7.2 (Centers for Disease Control, Atlanta, USA; https://www.cdc.gov/epiinfo/ index.html).

A pesquisa foi aprovada pelo Comitê de Ética em Pesquisa da instituição (CAAE no 24819013100005274$)$.

\section{RESULTADOS}

Participaram do estudo 46 residentes no primeiro (T1), 42 no segundo (T2) e 40 no terceiro (T3) momento. As perdas de seguimento ocorreram por motivos de afastamento, desistência ou desligamento. Os dados sociodemográficos dos participantes no início do programa (T1) estão descritos na Tabela 1 


\begin{tabular}{|c|c|c|}
\hline \multicolumn{3}{|c|}{$\begin{array}{c}\text { TABELA } 1 \\
\text { Dados sociodemográficos dos residentes } \\
\text { ao início do programa (T1). }\end{array}$} \\
\hline & & n (\%) \\
\hline \multirow[t]{3}{*}{ IDADE } & Até 25 & $31(67,39)$ \\
\hline & Entre 26 e 30 & $14(30,43)$ \\
\hline & Acima de 30 & $1(2,17)$ \\
\hline \multirow[t]{2}{*}{ GENERO } & Feminino & $36(78,26)$ \\
\hline & Masculino & $10(21,74)$ \\
\hline \multirow[t]{2}{*}{ ESTADO CIVIL } & Solteiro(a) & $42(91,30)$ \\
\hline & Casado(a) & $4(8,70)$ \\
\hline \multirow[t]{2}{*}{ FILHOS } & Não & $44(95,65)$ \\
\hline & Sim & $2(4,35)$ \\
\hline \multirow[t]{2}{*}{ SITUAÇÃO DE MORADIA } & Alojamento & $5(10,87)$ \\
\hline & $\begin{array}{l}\text { Casa própria } \\
\text { ou família }\end{array}$ & $41(89,13)$ \\
\hline \multirow{3}{*}{$\begin{array}{l}\text { PRINCIPAL MEIO } \\
\text { DE TRANSPORTE }\end{array}$} & A pé & $9(19,57)$ \\
\hline & Transporte coletivo & $33(71,74)$ \\
\hline & Transporte próprio & $4(8,70)$ \\
\hline \multirow[t]{3}{*}{ TEMPO DE LOCOMOÇÃO } & Até $30 \mathrm{~min}$ & $18(39,13)$ \\
\hline & De 31 a $60 \mathrm{~min}$ & $11(23,91)$ \\
\hline & Acima de $61 \mathrm{~min}$ & $17(36,96)$ \\
\hline \multirow[t]{8}{*}{ CATEGORIA PROFISSIONAL } & Enfermagem & $14(30,43)$ \\
\hline & Farmácia & $5(10,87)$ \\
\hline & Física Médica & $5(10,87)$ \\
\hline & Fisioterapia & $5(10,87)$ \\
\hline & Nutrição & $5(10,87)$ \\
\hline & Odontologia & $2(4,35)$ \\
\hline & Psicologia & $4(8,70)$ \\
\hline & Serviço Social & $6(13,04)$ \\
\hline \multirow[t]{3}{*}{ TEMPO DE FORMADO } & Menos que dois & $37(80,43)$ \\
\hline & Entre dois e quatro & $7(15,22)$ \\
\hline & Cinco ou mais & $2(4,35)$ \\
\hline
\end{tabular}

Ao longo do programa foi identificado que houve aumento do número de residentes que passaram a residir no alojamento, sendo $52,38 \%$ no T2 e 60,00\% no T3, e consequente diminuição do tempo de locomoção, quando a maioria passou a necessitar de menos de 30 minutos para chegar até os cenários de aprendizagem da residência, sendo 57,14\% no T2 e 70,00\% no T3.

A maioria dos residentes possuía algum hobby, não praticava atividade física, não fumava e não teve afastamento por motivos de saúde. $\mathrm{O}$ consumo de bebida alcoólica apresentou uma discreta redução em relação ao momento de entrada no curso e o número de residentes que tiveram afastamento por motivos de saúde aumentou. Houve aumento da intensidade da EE e da baixa RP, tendo, como consequência, o aumento da frequência da síndrome de burnout ao longo do programa. Também houve aumento da frequência e da intensidade de depressão (Tabela 2).
TABELA 2

Hábitos pessoais, afastamento por motivos de saúde e prevalência de burnout e de depressão em residentes ao longo de um programa multiprofissional em oncologia

\begin{tabular}{|c|c|c|c|c|}
\hline & & $\begin{array}{c}\mathrm{T} 1=46 \\
\mathrm{n}(\%)\end{array}$ & $\begin{array}{c}\mathrm{T} 2=42 \\
\text { n }(\%)\end{array}$ & $\begin{array}{c}\text { T3 }=40 \\
\text { n }(\%)\end{array}$ \\
\hline \multicolumn{5}{|l|}{ НОВВУ } \\
\hline \multicolumn{2}{|l|}{ Não } & $14(30,43)$ & $8(19,05)$ & $12(30,00)$ \\
\hline \multicolumn{2}{|l|}{ Sim } & $32(69,57)$ & $34(80,95)$ & $28(70,00)$ \\
\hline \multicolumn{5}{|l|}{ ATIVIDADE FISICA } \\
\hline \multicolumn{2}{|l|}{ Não } & $32(69,57)$ & $30(71,43)$ & $27(67,50)$ \\
\hline \multicolumn{2}{|l|}{ Sim } & $14(30,43)$ & $12(28,57)$ & $13(32,50)$ \\
\hline \multicolumn{5}{|l|}{ TABAGISMO } \\
\hline \multicolumn{2}{|l|}{ Não } & $45(97,83)$ & $42(100,00)$ & $39(97,50)$ \\
\hline \multicolumn{2}{|l|}{ Sim } & $1(2,17)$ & $0(0,00)$ & $1(2,50)$ \\
\hline \multicolumn{5}{|l|}{$\begin{array}{l}\text { CONSUMO DE } \\
\text { BEBIDA ALCÓOLICA }\end{array}$} \\
\hline \multicolumn{2}{|l|}{ Não } & $23(50,00)$ & $26(61,90)$ & $23(57,50)$ \\
\hline \multicolumn{2}{|l|}{ Sim } & $23(50,00)$ & $16(38,10)$ & $17(42,50)$ \\
\hline \multicolumn{5}{|l|}{$\begin{array}{l}\text { AFASTAMENTO POR } \\
\text { MOTIVO DE SAÚDE }\end{array}$} \\
\hline \multicolumn{2}{|l|}{ Não } & $41(89,13)$ & $35(83,33)$ & $27(67,50)$ \\
\hline \multicolumn{2}{|l|}{ Sim } & $5(10,87)$ & $7(16,67)$ & $13(32,50)$ \\
\hline \multicolumn{5}{|l|}{ BURNOUT } \\
\hline \multirow{3}{*}{$\begin{array}{l}\text { Exaustão } \\
\text { emocional }\end{array}$} & Alto & $8(17,39)$ & $15(35,71)$ & $30(75,00)$ \\
\hline & Intermediário & $6(13,04)$ & $15(35,71)$ & $5(12,50)$ \\
\hline & Baixo & $32(69,57)$ & $12(28,57)$ & $5(12,50)$ \\
\hline \multirow[t]{3}{*}{ Despersonificação } & Alto & $9(19,57)$ & $8(19,05)$ & $6(15,00)$ \\
\hline & Intermediário & $6(13,04)$ & $8(19,05)$ & $9(22,50)$ \\
\hline & Baixo & $31(67,39)$ & $26(61,90)$ & $25(62,50)$ \\
\hline \multirow{3}{*}{$\begin{array}{l}\text { Baixa } \\
\text { realização pessoal }\end{array}$} & Alto & $13(28,26)$ & $19(45,24)$ & $16(40,00)$ \\
\hline & Intermediário & $14(30,43)$ & $16(38,10)$ & $16(40,00)$ \\
\hline & Baixo & $19(41,30)$ & $7(16,67)$ & $8(20,00)$ \\
\hline \multirow{2}{*}{$\begin{array}{l}\text { Síndrome } \\
\text { de burnout }\end{array}$} & Não & $33(71,74)$ & $22(52,38)$ & $10(25,00)$ \\
\hline & Sim & $13(28,26)$ & $20(47,62)$ & $30(75,00)$ \\
\hline \multirow[t]{4}{*}{ DEPRESSÃO } & Grave & $0(0,00)$ & $1(2,38)$ & $1(2,50)$ \\
\hline & $\begin{array}{l}\text { Moderada a } \\
\text { grave }\end{array}$ & $1(2,17)$ & $3(7,14)$ & $7(17,50)$ \\
\hline & $\begin{array}{l}\text { Leve a } \\
\text { moderada }\end{array}$ & $7(15,22)$ & $17(40,48)$ & $21(52,50)$ \\
\hline & $\begin{array}{l}\text { Sem ou } \\
\text { mínima }\end{array}$ & $38(82,61)$ & $21(50,00)$ & $11(27,50)$ \\
\hline
\end{tabular}

T1 - início do programa; T2- Termino do primeiro ano; T3 - Término do segundo ano. *Alto nível de Exaustão emocional e/ou Despersonificação

Verificou-se a ocorrência de síndrome de burnout e de depressão ao longo do programa (T2 e T3) em profissionais residentes de todas as categorias profissionais. Não foi identificada correlação entre os hábitos pessoais e a ocorrência de síndrome de burnout ou depressão. Observou-se uma correlação positiva entre o afastamento por motivos de saúde com a presença de síndrome de burnout no segundo momento $(\mathrm{p}=0,03)$ e com depressão nos segundo $(p=0,05)$ e terceiro $(p=0,05)$ momentos. 
A síndrome de burnout esteve correlacionada com ocorrência de depressão no primeiro $(p=0,03)$ e no terceiro $(p=0,01)$ momento.

Ao longo do programa houve aumento significativo da ocorrência de síndrome de burnout entre T1 e T2 $(\mathrm{p}=0,05), \mathrm{T} 1$ e T3 ( $p=<0,01)$ e T2 e T3 ( $p=0,01)$. Também foi encontrado aumento significativo dos casos de depressão entre T1 e T2 ( $\mathrm{p}=$ $<0,01)$, T1 e T3 $(\mathrm{p}=<0,01)$ e T2 e T3 $(\mathrm{p}=0,03)$.

Houve aumento significativo da EE no final do primeiro (T2) e do segundo (T3) ano em relação ao momento de ingresso no programa (T1), assim como aumento significativo entre o final do programa (T3) e o final do primeiro ano (T2). Observou-se, também, incremento significativo da baixa RP entre os residentes no final do primeiro (T2) e do segundo (T3) ano, em relação ao momento de ingresso no programa (T1). A intensidade de depressão aumentou no final do primeiro (T2) e do segundo (T3) ano, em relação ao momento de ingresso no programa (T1) (Tabela 3).
A dinâmica dos programas de residência traz uma mudança brusca no cotidiano dos profissionais de saúde, que transitam da condição de estudante para a de trabalhador estudante, com consequente aumento de responsabilidades e conflitos. A extensa carga horária semanal para cumprimento do programa envolve estratégias educacionais teóricas, teórico-práticas e práticas que exigem um alto nível de atenção, dedicação e comprometimento ${ }^{18}$. Tais aspectos promovem um ambiente colaborativo para a ocorrência dos problemas identificados. Fatores do ambiente de aprendizagem e de trabalho, ao invés de atributos individuais, têm sido destacados como os principais fatores de desgaste de residentes ${ }^{6,9}$.

A concepção pedagógica prevista para os programas de residência envolve a utilização de metodologias ativas, problematização e abordagem multiprofissional. Esse desenho, em geral, é inovador para os recém-graduados, promovendo certo tensionamento no processo de aprendizagem ${ }^{19}$. Destarte,

\begin{tabular}{|c|c|c|c|c|c|c|}
\hline \multicolumn{7}{|c|}{$\begin{array}{l}\text { TABELA } 3 \\
\text { as dos scores dos componentes da síndrome } \\
\text { essão entre os momentos estudados. }\end{array}$} \\
\hline & $\mathrm{T} 1=46$ & $\mathrm{~T} 2=42$ & $\mathrm{~T} 3=40$ & $\mathrm{~T} 1 / \mathrm{T} 2$ & $\mathrm{~T} 1 / \mathrm{T} 3$ & T2 / T3 \\
\hline & $\begin{array}{l}\text { Mediana } \\
\text { (max-min) }\end{array}$ & $\begin{array}{l}\text { Mediana } \\
\text { (max-min) }\end{array}$ & $\begin{array}{l}\text { Mediana } \\
\text { (max-min) }\end{array}$ & p valor* & p valor* & p valor* \\
\hline \multicolumn{7}{|l|}{ BURNOUT } \\
\hline Exaustão emocional & $13,5(0-35)$ & $24,0(6-38)$ & $31,5(6-48)$ & $<0,01$ & $<0,01$ & $<0,01$ \\
\hline Despersonificação & $3,0(0-18)$ & $4,0(0-19)$ & $3,5(0-27)$ & 0,56 & 0,40 & 0,80 \\
\hline Baixa realização pessoal & $38,0(25-47)$ & $35,0(20-46)$ & $34,5(20-44)$ & 0,03 & 0,01 & 0,74 \\
\hline DEPRESSÃO & $6,0(0-28)$ & $9,5(2-35)$ & $11,0(0-36)$ & $<0,01$ & $<0,01$ & 0,13 \\
\hline
\end{tabular}

*Teste de Wilcoxon.

T1 - início do programa; T2- Termino do primeiro ano; T3 - Término do segundo ano.

\section{DISCUSSÃO}

O presente estudo revelou uma alta prevalência e aumento da incidência de síndrome de burnout e de depressão entre profissionais de saúde ao longo de dois anos de um programa de residência multiprofissional em oncologia. Além disso, foi identificada forte correlação entre a ocorrência dos dois problemas de saúde entre os investigados.

Em sua maioria os residentes eram jovens e recém-formados. A baixa média etária e a pouca experiência profissional podem afetar diretamente a forma pela qual o residente enfrenta os desafios intrínsecos aos programas de residência ${ }^{18}$, principalmente na área de oncologia, tão relacionada a tratamentos complexos e à finitude. Somam-se a isso as ansiedades relativas à escolha profissional, o estresse inerente ao término da graduação e as incertezas relacionadas à inserção no mercado de trabalho. o estímulo ao desenvolvimento da autonomia, necessária aos discentes, conflita com a constante necessidade de supervisão por parte do corpo docente-assistencial dos programas, o que acarreta em uma certa dualidade de identidade (estudante-trabalhador), que pode comprometer o estado emocional.

Ao final do programa $60,00 \%$ dos residentes moravam no alojamento da instituição. Embora a oferta de alojamento possa viabilizar a realização do programa de residência, principalmente àqueles que moram em outros municípios ou estados da federação, reduzindo o tempo de locomoção até os cenários de aprendizagem, não se pode negligenciar o fato dos profissionais se manterem distantes de familiares e amigos e próximos do ambiente da residência. Estes fatores podem contribuir para o adoecimento relacionado à essa modalidade de formação.

Ressalta-se, ainda, que foram identificados casos de síndrome de burnout e de depressão em residentes de todas as 
categorias profissionais ao longo do programa. Este achado, semelhante ao encontrado em recente estudo nacional ${ }^{20}$, pode indicar que os elementos relacionados aos problemas identificados não estão limitados à atuação específica de um núcleo profissional, mas aos aspectos estruturais que envolvem os programas de residência e o cuidado ao paciente oncológico.

A complexidade da atenção ao câncer, que envolve a noção de etiologia, fatores de risco, epidemiologia, prevenção, detecção precoce e as multimodalidades de tratamentos altamente especializados, exige dos profissionais a aquisição de conhecimentos inovadores, em geral, não apresentados durante a graduação. Isto exige um maior empenho por parte dos profissionais e traz novas exigências pessoais, podendo contribuir para a exaustão emocional.

Durante o desenvolvimento do programa é necessário, ainda, que os residentes desenvolvam, em um curto período de tempo, um conjunto de habilidades específicas para lidar com o paciente oncológico. A alta criticidade do câncer e as fragilidades dos indivíduos, por vezes agravadas pelo próprio tratamento, traz desafios adicionais aos profissionais, que se sentem inseguros quanto a sua atuação, podendo trazer sensação de impotência, fracasso e baixa realização pessoal ${ }^{21}$.

Agrega-se as dificuldades individuais em enfrentar frequentes situações que envolvam dor, sofrimento, morte e luto, principalmente considerando a idade e a pouca experiência profissional dos residentes. Aspectos subjetivos relacionados ao lidar com estas situações, como identificação e transferência, também podem colaborar para o desenvolvimento de desgaste físico e emocional.

Outro dado que pode ser destacado é a relação existente entre o afastamento por motivo de saúde e a ocorrência de síndrome de burnout e depressão. A identificação dessa correlação aponta para a gravidade da situação, considerando que o pouco tempo de exercício profissional na área da saúde parece ser suficiente para o desenvolvimento dos problemas em questão. Ressalta-se, ainda, que o absenteísmo ao proporcionar interrupções no processo formativo pode comprometer a aprendizagem.

É interessante apontar que o oposto do absenteísmo, ou seja, o presenteísmo também pode aumentar os níveis de burnout ${ }^{22}$. Trata-se de um fenômeno em que os trabalhadores permanecem no trabalho mesmo estando doentes ${ }^{19}$. No caso da residência, a obrigatoriedade de cumprimento de toda a carga horária prática do programa, para que se consiga a certificação, e o excesso de pressão pelo corpo docente-assistencial podem favorecer esse tipo de atitude, prejudicar a aprendizagem do residente e colocar em risco a qualidade da assistência prestada aos pacientes.
Em relação aos componentes do burnout, destacam-se os níveis crescentes da frequência e da intensidade de EE ao longo de todo o programa de residência. Tal fato pode estar relacionado ao acúmulo de atividades propostas nos programas de residência, agravadas pelo desenvolvimento do trabalho de conclusão da residência e pela expectativa de realização de procedimentos mais complexos e com maior autonomia.

A frequência e intensidade de baixa RP aumentou do momento do ingresso até o final do primeiro ano e se manteve elevada até o final do segundo ano. É possível que essa condição esteja relacionada a uma certa sensação de impotência diante da resolutividade de determinadas situações, considerando o momento de inserção no campo de atividades práticas e a ampliação dos conhecimentos sobre as dificuldades do enfrentamento do câncer, em termos técnico-científicos, político-gerenciais-estruturais e emocionais. Além disso, as dúvidas sobre a escolha de atuação profissional, a incerteza sobre o futuro e as situações de sofrimento vivenciadas durante a residência também podem contribuir para esse atributo ${ }^{18,20,23}$.

Destaca-se que não foram observadas mudanças significativas na frequência ou intensidade da DP ao longo do programa. Tendo em vista que este componente está relacionado a insensibilidade e a indiferença nas relações interpessoais, acredita-se que a concepção de humanização, preconizada pelas políticas de saúde vigentes e incorporadas à grade curricular dos cursos de graduação na área da saúde e nos programas de residência, possa estar se refletindo nos cotidianos do cuidado em saúde. Outro elemento que pode contribuir para este resultado é a área de concentração do programa, haja vista que o câncer e o contato cotidiano com o sofrimento podem contribuir para um ambiente mais altruísta.

A depressão foi mais frequente e intensa no final do primeiro e do segundo ano quando comparado ao momento inicial do programa, atingindo $72,5 \%$ dos residentes. Poderíamos supor que o treinamento em serviço, próprio da residência multiprofissional em oncologia, poderia justificar a alta frequência de depressão na amostra estudada. A depressão é uma condição presente durante os anos de treinamento de residentes $^{24}$. A sistematização das atividades desenvolvidas durante a residência, os cenários de prática e as dissonâncias existentes entre teoria e prática têm sido associados ao desenvolvimento de ansiedade e depressão ${ }^{25,26}$.

A depressão diminui a habilidade cognitiva e prejudica os cuidados e a segurança do paciente. Destaca-se que a depressão grave pode ser considerada uma doença que ameaça a vida, especialmente se não diagnosticada e tratada ${ }^{12}$.

Observou-se uma correlação positiva entre a ocorrência de síndrome de burnout com os casos de depressão. A idade 
dos sujeitos da presente pesquisa pode explicar a alta frequência tanto de burnout quanto de depressão, uma vez que jovens portadores de burnout apresentam maior frequência de depressão leve, quando comparado a ausência de depressão ${ }^{27}$. Outra possibilidade de explicação seria a comprovada predisposição à depressão, refletida pela história pessoal e familiar, relacionada ao aumento do risco de burnout ${ }^{28}$. Ademais, o estresse originado pelo acúmulo de atividades da residência pode provocar tanto o burnout quanto a depressão ${ }^{26}$.

Os resultados desse estudo são preocupantes sob os aspectos da educação, da saúde do trabalhador e do cuidado prestado aos usuários, tendo em consideração que os problemas identificados podem comprometer a aprendizagem, desestimular a escolha da especialidade, levar à desistência do programa, reduzir o prazer com a carreira escolhida, comprometer a qualidade de vida, favorecer erros e diminuir a qualidade da assistência ${ }^{20,26,29,30}$.

Neste sentido, essas condições não podem ser negligenciadas e demandam ações para a prevenção e o controle, considerando que as suas causas são multifatoriais e que quanto maior a exposição dos indivíduos, maior será a chance de desenvolvimento de ambos os problemas ${ }^{19,20}$. Cabe aos núcleos docente-estruturantes dos programas de residência de cada instituição identificar as causas e implementar estratégias para enfrentamento dessas situações ${ }^{20,23}$.

Apesar de haver questões estruturais inerentes aos programas de residência multiprofissionais em oncologia identificadas como fatores causais de síndrome de burnout e depressão, que estão fora da autonomia das coordenações dos programas, possíveis intervenções podem ser empregadas para o manejo dessas condições, tais como: proporcionar espaços de diálogo entre os atores dos programas de residência e serviços de saúde, estimular medidas de gestão do estresse ocupacional, oferecer reabilitação profissional, aperfeiçoar o processo de ensino-aprendizagem, desenvolver programas de apoio aos residentes e realizar treinamento para comunicação de notícias difíceis ${ }^{9,11,13,23,26,29-32}$.

Como limitações do estudo aponta-se que: os dados foram auto relatados, não tendo sido realizado nenhum exame diagnóstico para confirmação dos referidos problemas de saúde; o estudo envolveu residentes de uma única instituição e todos eram de uma mesma turma, não sendo possível realizar generalização dos achados; e, os fatores relacionados ao local da aprendizagem podem ter contribuído para os desfechos encontrados. No entanto, o método empregado no estudo é internacionalmente utilizado para este tipo de investigação e apesar das condições locais, os problemas estruturais impactam os programas de residência em todas as instituições. Cabe ressaltar que este foi o primeiro estudo a analisar a ocorrência de burnout e depressão em um programa de residência multiprofissional em oncologia.

\section{CONCLUSÃO}

O estudo revelou um alto nível de síndrome de burnout e de depressão em discentes de um programa de residência multiprofissional em oncologia. Os achados apontam para a gravidade do problema, considerando que todas as categorias profissionais foram afetadas e que ambas as condições aparecem ainda no primeiro ano de curso. Estratégias para prevenção e controle são necessárias, a fim de minimizar as consequências na aprendizagem, na qualidade de vida dos estudantes-trabalhadores e na assistência prestada aos pacientes com câncer.

\section{REFERÊNCIAS}

1. Brasil. Ministério da Saúde, Secretaria de Gestão do Trabalho e da Educação na Saúde, Departamento de Gestão da Educação na Saúde. Residência multiprofissional em saúde: experiências, avanços e desafios. 1ํ. ed. Brasília, DF; 2006. $414 \mathrm{p}$.

2. Brasil. Portaria Interministerial $\mathrm{n}^{\mathrm{o}}$ 16, de 22 de dezembro de 2014. Altera a Portaria Interministerial no 1.077/MEC/ MS, de 12 de novembro de 2009, a Portaria Interministerial no 1.320/MEC/MS, de 11 de novembro de 2010 e revoga a Portaria Interministerial no $\mathrm{n}^{\mathbf{0}}$ 1.224/MEC/MS, de 3 de outubro de 2012, para atualizar o processo de designação dos membros da Comissão Nacional de Residência Multiprofissional em Saúde (CNRMS) e para incluir áreas profissionais para a realização de Programas de Residência Multiprofissional e em Área Profissional da Saúde. Diário Oficial da União 2014;12 out.

3. Maslach C, Jackson SE. The measurement of experienced burnout. J Organ Behav 1981; 2(2):99-113.

4. Maslach C, Schaufeli WB, Leiter MP. Job Burnout. Annu Rev Psychol 2001; 52:397-422.

5. HahnMary K, Carlotto S. Síndrome de Burnout em monitores de uma fundação de proteção especial. Divers Perspect En Psicol 2008; 4(1):53-62.

6. Lima RAS, Souza AI, Galindo RH, Feliciano KVO. Vulnerability to burnout among physicians at a public hospital in Recife. Ciênc Saúde Coletiva 2013; 18(4):1051-1058.

7. Trufelli DC, Bensi CG, Garcia JB, Narahara JL, Abrão MN, Diniz RW, Miranda VC, Soares HP, Del Giglio A. Burnout in cancer professionals: a systematic review and meta-analysis. Eur J Cancer Care 2008; 17(6):524-531.

8. Tucunduva LTCM, Garcia AP, Prudente FVB, Centofanti G, Souza CM, Monteiro TA, Vince FAH, Samano EST, Gon- 
çalves MS, Giglio AD. A síndrome da estafa profissional em médicos cancerologistas brasileiros. Rev Assoc Med Bras 2006; 52(2):108-112.

9. Dyrbye L, Shanafelt T. A narrative review on burnout experienced by medical students and residents. Med Educ 2016; 50(1):132-149.

10. Franco GP, Barros ALBL, Nogueira-Martins LA, Zeitoun SS. Burnout em residentes de enfermagem. Rev Esc Enferm USP 2011; 45(1):12-18.

11. World Health Organization. Group Interpersonal Therapy (IPT) for depression. Geneva: WHO; 2016. 100 p.

12. Oliveira GS, Chang R, Fitzgerald PC, Almeida MD, Castro-Alves LS, Ahmad S, McCarthy RJ. The Prevalence of Burnout and Depression and Their Association with Adherence to Safety and Practice Standards: A Survey of United States Anesthesiology Trainees. Anesth Analg 2013; 117(1):182-193.

13. Zorzanelli R, Vieira I, Russo JA. Diversos nomes para o cansaço: categorias emergentes e sua relação com o mundo do trabalho. Interface - Comun Saúde Educ 2016; 20(56):77-88.

14. Trigo TR. Validade fatorial do Maslach Burnout Inventory-Human Services Survey (MBI-HSS) em uma amostra brasileira de auxiliares de enfermagem de um hospital universitário: influência da depressão [Internet]. Universidade de São Paulo; 2010 [acessado 2016 Dec 28]. Disponível em: http: / / www.teses. usp.br/teses/disponiveis/5/5142/tde-26052011-123120/ en.php

15. Cubero DIG, Fumis RRL, Sá TH, Dettino A, Costa FO, Van Eyll BMRHA, Beato C, Peria FM, Mota A, Altino J, Aevedo SJ, Rocha Filho DR, Moura M, Lessa AER, Giglio A. Burnout in Medical Oncology Fellows: a Prospective Multicenter Cohort Study in Brazilian Institutions. J Cancer Educ 2016; 31(3):582-587.

16. Beck AT. Psychometric properties of the Beck Depression Inventory: twenty-five years of evaluation. Clin Psychol Rev 1988; 8:77-100.

17. Gorenstein C, Andrade L. Inventário de depressão de Beck: propriedades psicométricas da versão em português. Rev Psiq Clin 1998; 25(5):245-250.

18. Guido LA, Silva RM, Goulart CT, Bolzan MEO, Lopes LFD. Síndrome de Burnout em residentes multiprofissionais de uma universidade pública. Rev Esc Enferm USP 2012; 46(6):1477-1483.

19. Guido LA, Goulart CT, Silva RM, Lopes LFD, Ferreira EM. Estresse e Burnout entre residentes multiprofissionais. Rev Lat-Am Enferm 2012; 20(6):1064-1071.

20. Sanches VS, Ferreira PM, Veronez AV, Koch R, Souza AS, Cheade MFM, Christofoletti G. Burnout e Qualidade de
Vida em uma Residência Multiprofissional: um Estudo Longitudinal de Dois Anos. Rev Bras Educ Médica 2016; 40(3):430-436

21. Guveli H, Anuk D, Oflaz S, Guveli ME, Yildirim NK, Ozkan M, Ozkan S. Oncology staff: burnout, job satisfaction and coping with stress: Burnout of the oncology employees. Psychooncology 2015; 24(8):926-931.

22. Demerouti E, Le Blanc PM, Bakker AB, Schaufeli WB, Hox J. Present but sick: a three-wave study on job demands, presenteeism and burnout. Career Dev Int 2009; 14(1):50-68.

23. Fernandes MNS, Beck CLC, Weiller TH, Viero V, Freitas $\mathrm{PH}$, Prestes FC. Sofrimento e prazer no processo de formação de residentes multiprofissionais em saúde. Rev Gaúcha Enferm 2015; 36(4):90-97.

24. Goebert D, Thompson D, Takeshita J, Beach C, Bryson P, Ephgrave K, Kent A, Kunkel M, Schechter J, Tate J. Depressive symptoms in medical students and residents: a multischool study. Acad Med 2009; 84(2):236-241.

25. Rotta DS, Pinto MH, Lourenção LG, Teixeira PR, Gonsalez EG, Gazetta CE. Anxiety and depression levels among multidisciplinary health residents. Rev Rede Enferm Nordeste 2016; 17(3):372-377.

26. Silva RM, Batista KM, Serrano PM, Lopes LFD, Costa ALS, Guido LA. Influência do Hardiness no Burnout e depressão entre residentes multiprofissionais- estudo analítico. Rev Enferm Atual Derme 2014; 71:18-22.

27. Iacovides A, Fountoulakis K, Kaprinis S, Kaprinis G. The relationship between job stress, burnout and clinical depression. J Affect Disord 2003; 75(3):209-221.

28. Nyklí $\square$ ek I, Pop VJ. Past and familial depression predict current symptoms of professional burnout. J Affect Disord 2005; 88(1):63-68.

29. Blanchard P, Truchot D, Albiges-Sauvin L, Dewas S, Pointreau Y, Rodrigues M, Xhaard A, Loriot Y, Giraud P, Soria JC, Kantor G. Prevalence and causes of burnout amongst oncology residents: A comprehensive nationwide cross-sectional study. Eur J Cancer 2010; 46(15):2708-2715.

30. McCray LW, Cronholm PF, Bogner HR, Gallo JJ, Neill RA. Resident physician burnout: is there hope? Fam Med 2008; 40(9):626-632.

31. Bragard I, Etienne A-M, Libert Y, Merckaert I, Liénard A, Meunier J, Delvaux N, Hansez I, Marchal S, Reynaert C, Slachmuylder JL, Ravazi D. Predictors and Correlates of Burnout in Residents Working With Cancer Patients. J Cancer Educ 2010; 25(1):120-126.

32. Moody K, Kramer D, Santizo RO, Magro L, Wyshogrod D, Ambrosio J, Castillo C, Lieberman R, Stein J. Helping the Helpers Mindfulness Training for Burnout in Pediatric 
Oncology-A Pilot Program. J Pediatr Oncol Nurs 2013; 30(5):275-284.

\section{CONTRIBUIÇÃO DOS AUTORES}

Ismar Lima Cavalcanti contribuiu na concepção, análise e interpretação dos dados e na redação do artigo, Fernando Lopes Tavares de Lima e Mario Jorge Sobreira da Silva contribuíram no delineamento do estudo, análise e interpretação dos dados e redação do artigo, e Telma de Almeida Souza participou no delineamento da pesquisa e revisão crítica do artigo. Todos os autores aprovaram a versão final encaminhada.

\section{CONFLITO DE INTERESSES}

Nenhum há declarar

\section{ENDEREÇO PARA CORRESPONDÊNCIA}

Ismar Lima Cavalcanti

Endereço: Rua Marquês de Pombal, 125 - $3^{\circ}$ andar - Centro

Rio de Janeiro - RJ - CEP: 20230-240 Brasil 